\section{Victoria Forester Wins International Award}

Dr. Allan Van Sickle, a forest pathologist with the Pacific Forest Research Centre, Victoria, B.C., was awarded the Schlich Memorial Prize during a recent sabbatical at the Oxford School of Forestry in England. The Schlich Award is named after Sir William Schlich, a world renowned forester, who founded the Forestry School at Oxford in 1905.

The prize, a bronze plaque and a set of books, is awarded individuals making the best use of their time while attending the Oxford Forestry School. Dr. Van Sickle was the Canadian participant in the Planning and Management course sponsored by the Commonwealth Forestry Institute. This course is given every two years for foresters from countries throughout the world.

It is notable that in $1965 \mathrm{Dr}$. Van Sickle won the Schlich Canadian Award while attending the University of British Columbia.

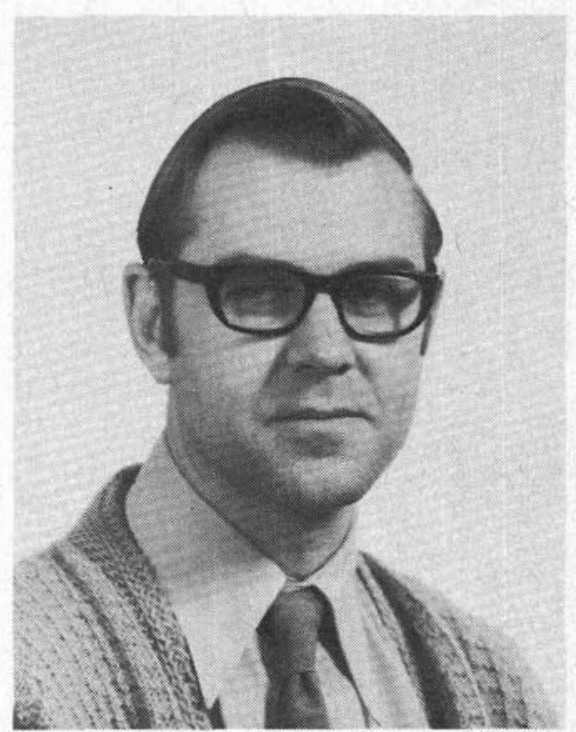

Allan Van Sickle

\section{New Members}

\section{Active}

N. E. Alexander

6623 - 19 St. - R.R. 9

Surrey, B.C. V3S 5M1

T. M. Apsey

3824 Campus Cres.

Victoria, B.C. V8P $2 W 9$

G. C. Archekin

1070 Nanton Ave.

Vancouver, B.C. V6H $2 \mathrm{C} 2$

N. P. Balaski

c/o Procter \& Gamble Cellulose

Postal Bag 1030

Grande Prairie, Alta

V. E. Bradford

Gen. Del.

Ignace, Ont. POT 1TO

B. E. Firby

888 - 29th. St. E.

Prince Albert, Sask. S6V 1Y1

Claude Gignac

38 St. Donat

Ste. Agathe des Monts, P.Q.

J8C 1 P8

C. G. Griffis

327 Otto St. Apt \# 2

Thunder Bay, Ont. P7A 2V2

B. D. Heine

912 Mapledale Place

Calgary, Alta T2J 1W6

J. S. Hermelin

430 Lisgar

Fredericton, N.B. E3B 3B2

D. F. Johnson

525 Herbert St.

Pembroke, Ont. K8A 2Z4
B. Juneau

656 De Lestre Apt. \# 1

Ste Foy, Que. G1X 2L8

K. E. Reid

113 Crocket St.

Fredericton, N.B. E3A $4 \mathrm{H} 2$

Janet Schilf

10512-70th. Ave.

Edmonton, Alta T6H 2E9

W. T. Wachsmuth

37 Poplar St.

Chatham, Ont. N7L 1J8

R. C. West

Dept. Natural Resources

Box 6000

Fredericton, N.B. E3B $5 \mathrm{H} 1$

W. S. Wilmot

35 Ivy Crescent

Ottawa, Ont. K1M 1Y1

\section{Affiliate}

H. L. Murdoch

32913 - Cardinal St.

R.R. \# 2. Mission, B.C.

\section{A. G. Newbert}

Box 488

Tahsis, B.C. VOP $1 \times 0$

M. Y. Wai

Strategic Studies

Min. of Forests - Legis. Bldg.

Victoria, B.C. V8W 3E7

H. J. Whittaker

831 - 355 Burrard St.

Vancouver, B.C. V6C $2 \mathrm{H} 1$

\section{Student}

Robert Anderson

7319 - 113 St.

Edmonton, Alta
G. D. Arthurs

Box 39 - Site 2 RR. \#3

New Maryland, N.B. E3B 4 X6

P. S. Babor

85 Bedford Rd.

Toronto, Ont. M5R 2K3

B. R. Beaton

Camp 2 - Site 5 - RR. \#3

Fredericton, N.B. E3B 4X4

Sherrylynn H. Boycott

Lakehead Univ. Residence

Thunder Bay, Ont. P7B 5E1

Cindy Brereton

23 Westmount Park Rd.

Weston, Ont. M9P 1R4

Judy A. Briand

530 Needham St.

Fredericton, N.B.

B. Callaghan

50 Glengowan Rd.

Toronto, Ont. M4N 1 G2

I. R. Cameron

3970 W. - 19th

Vancouver, B.C.

G. E. Caron

647 Scully St.

Fredericton, N.B. E3B 1V3

Deborah Cermak

70 Cockburn Dr.

West Hill, Ont.

L. G. Competrin

470 Kings College Rd.

Fredericton, N.B. E3B 2 G5

S. J. Conn

Camp 2 - Site 5 - RR \#3

Fredericton, N.B. E3B $4 \mathrm{X} 4$

G. A. Cowan

L. U. Residence

Lakehead Univ.

Thunder Bay, Ont. P7B 5E1 
R. D. Copithorne

$10540-127$ St.

Edmonton, Alta. T5N 1V9

Lucy M. Czerwinski

192 Clinton St.

Toronto, Ont. M6G 2Y5

P. F. Dennison

30 Doerr Rd.

Scarborough, Ont. M1P 3A1

C. B. Duane

10942 University Ave.

Edmonton, Alta. T6G 1Y2

J. M. Duncan

304 Bridges House

U.N.B.

Fredericton, N.B.

Susan Dunn

77 N. Hill St.

Thunder Bay, Ont. P7A 5V5

R. Dunnigan

10969 - 131 St.

Edmonton, Alta T5H 1 B 7

J. A. Dunster

Faculty of Forestry - UBC

2075 Wesbrook PI.

Vancouver, B.C. V6T 1W5

Karl Dyer

607 Bridge St. E.

Belleville, Ont. K8N 5G9

Pulie Essau

83 Peter St.

Thunder Bay, Ont. P7A 5H4

I. C. G. Finlay

Lakehead Univ. Residence

Thunder Bay, Ont. F7B 5E1

J. M. Finstad

Lakehead Univ. Residence

Thunder Bay, Ont. F7B 5E1

R. J. Frech

Box $76-21$ Classic Ave.

New College, Ont.

Karen Fried

Lakehead Univ.

Thunder Bay, Ont. P7B 5E1

F. Gebbink

R.R. \#4

Red Deer, Alta T4N 5E4

M. C. Gibson

32 Aloma Cres.

Brampton, Ont. L6T 2P1

D. E. Grant

351 Osiris Dr.

Richmond Hill, Ont.

Sybille Haeussler

4623 - W. 12th.

Vancouver, B.C. V6P $2 \mathrm{R} 7$

J. E. Hall

40 Chipper Cres.

Scarborough, Ont. M1K 4R3

W. C. Hewitt

Box $99-21$ Classic Ave.

Toronto, Ont.

N. R. Hunter

10730 - 104th St.

Edmonton, Alta T5H 2W5
R. C. Ingersoll

73 Kent St.

Fredericton, N.B. E3A 4 Z8

P. Janas

287 Belsize Dr.

Toronto, Ont. M4S 1M5

L. B. Kaseram

30 Charles St. W. - Apt. 1222

Toronto, Ont. M4Y 1R5

\section{E. Kloss}

Lakehead Univ. Residence

Thunder Bay, Ont. F7B 5E1

G. Koopman

945 Dalhousie Dr.

London, Ont. N6K $1 \mathrm{M} 8$

Sally Krigstin

124 Sandringham Dr.

Downsview, Ont. M3H 1E3

A. C. Kwok Keung

161 Madison Ave.

Toronto, Ont. M5R 2S6

K. V. Lavelle

\# 107 - 11455 - 41 Ave.

Edmonton, Alta

Wendy J. Leclair

1166 Stream Bank Dr.

Mississauga, Ont. L5H 1W8

K. M. Lennon

105 Cornelius Parkway

Toronto, Ont. M6L 2K6

Michael Lipa

Lakehead Univ. Residence

Thunder Bay, Ont. F7A 5Y8

J. G. Long

R.R. \# 1

Trout Creek, Ont.

Janet Lyon

R.R. \# 4

Kemptiville, Ont. K0B 1J0

Susan Macbrien

Lakehead Univ. Residence

Thunder Bay, Ont. P7B 5E1

G. L. MacDougall

Box 1478

Kindersley, Sask. SoL 1S0

R. W. Mackay

10576 - 1 Ave.

Edmonton, Alta

D. A. MacLeod

721 Forest Hill Rd.

Fredericton, N.B. E3B $4 \mathrm{~K} 9$

G. Brian Maier

14423 - 78 Ave

Edmonton, Alta T5R 3C2

Andre Mallette

200 Hillview

Gatineau, P.Q. J8P 2L8

J. W. McCarthy

647 Windsor St.

Fredericton, N.B. E3B 4G3

Heather McCaw

85 St. George St. - Apt. 364

Toronto, Ont. M5S 2E5
G. I. McCormack

c/o Lakehead Univ. Residence

Thunder Bay, Ont. P7B 5E1

J. McNeil

370 St. Clements Ave.

Toronto, Ont. M5N 1M1

D. G. Millson

77 N. Hill St.

Thunder Bay, Ont. P7A 5V5

Sarah Moore

1457 Kalligan Crt.

Mississauga, Ont. L4X 1A9

Diana J. Morgan

53 Surbray Grove

Mississauga, Ont. L5B 2E2

Sylvia Mulder

7606 - 87th. St.

Edmonton, Alta

R. J. Murphy

2130 W. 33 Rd.

Vancouver, B.C. V6H 1 B9

Anne E. Mustard

6819 - 111 St.

Edmonton, Alta T6H $3 \mathrm{G} 2$

E. Nakagawa

420 Westmount Ave.

Toronto, Ont. M6E 3N5

W. J. Parton

10 Marilake Dr.

Agincourt, Ont. M1S 1V7

Kim Plater

10922 - 65 Ave.

Edmonton, Alta T6H 4R6

K. Powell

71 Fortrore Crescent

Don Mills, Ont. M3A $2 \mathrm{H} 2$

D. W. Pyke

Lakehead Univ. Residence

Thunder Bay, Ont. F7B 5E1

J. C. Racz

49 Regal Rd.

Toronto, Ont.

E. M. Saagh

1220 Shaw St.

Toronto, Ont. M6G 3N6

Janice Ellen Schmidt

274 Sheldrake Blvd.

Toronto, Ont. M4P 2B6

J. P. Schueller

11 - 97 Stardust Cres.

Thunder Bay, Ont. P7A 6G7

R. Schumacher

3075 W. 5th.

Vancouver, B.C. V6K 1 T8

G. Shapendonk

4650 W. 9th Ave.

Vancouver, B.C. V6R 2E4

Fiona M. Simmonds

373 N. Brodie St.

Thunder Bay, Ont. F7C 3T3

Jane Lloyd-Smith

360 Church St.

Fredericton, N.B. E3B 5B2 
R. Snyder

120 Fortrose Cresc.

Don Mills, Ont.

R. Spaans

Lakehead Univ. Residence

Thunder Bay, Ont. F7B 5E1

R. J. Spence

14 Balaclaua Ds.

Scarborough, Ont.

P. Srivastava

31 Hill St.

West Montrose, Ont. NOB 2V0

Inga Stadus

103 E. 39th. St.

Hamilton, Ont. L8V $4 \mathrm{H} 2$

J. A. Steponas

310 Rathburn Rd.

Islington, Ont. M9B 2C8

M. J. Stevens

RM. D107 - L.U. Residence

Lakehead Univ.

Thunder Bay, Ont. P7B 5E1

G. E. Sykes

47 Major St.

Toronto, Ont.

S. P. Taylor

9837 - 93 Ave.

Edmonton, Alta T6E 3W1

L. H. Tuan

159 Beverley St.

Toronto, Ont. M5T 1Y7
Margaret Upton

YWCA, 80 Woodlaw Ave. E.

Toronto, Ont. M4T 1C1

E. H. Wilford

\# $10-821$ W. 10th.

Vancouver, B.C.

Dennis Young

7319 - 113 St.

Edmonton, Alta

Regina W. Zuwa

20 Dorward Dr.

Rexdale, Ont. H9V 2J6

P. E. Zundel

104 Marbury Cresc.

Don Mills, Ont. M3A 2G4

Mel Zwierink

\#17-8631 - 105 St.

Edmonton, Alta

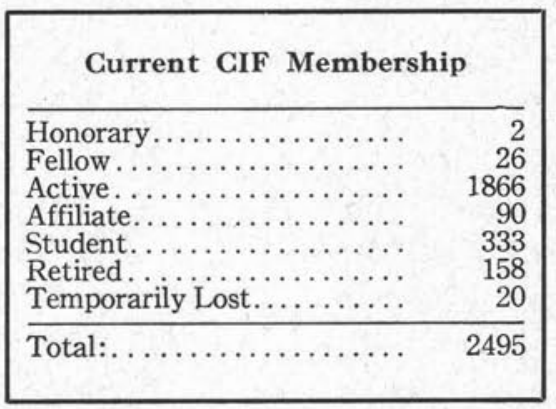

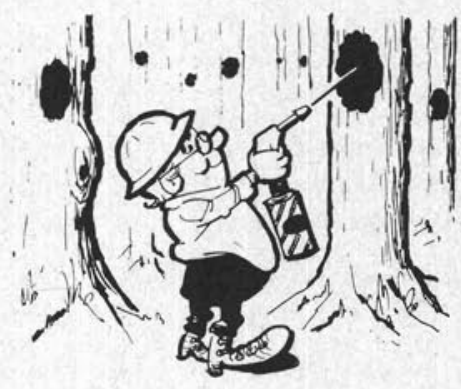

A logger named Paul couldn't see

The marks he had made on a tree.

After a lot of complaint he tried Nelson Paint

And now he is marking with glee!

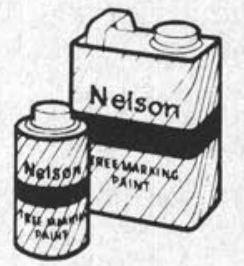

DOMINION CHAIN COMPANY Montreal, Que., Stratford, Ont., Vancouver, B.C.

\section{FACULTY POSITIONS IN FORESTRY \\ Faculty of Forestry and Landscape Architecture University of Toronto}

Full-time forestry faculty are required for July 1, 1979 in the following disciplines:

- SILVICULTURE

- TIMBER HARVESTING

- FOREST SOILS

- WOOD SCIENCE AND FOREST PRODUCTS

- FOREST ECOLOGY

- FOREST MANAGEMENT

Duties:

Teaching and research in forestry undergraduate and graduate programs.

Qualifications:

Ph.D. or equivalent experience, with one degree in forestry.

Recent and current Ph.D. graduates are encouraged to apply.

Salary and academic rank commensurate with qualifications and experience.

Send curriculum vitae, transcripts, names of three professional references to:

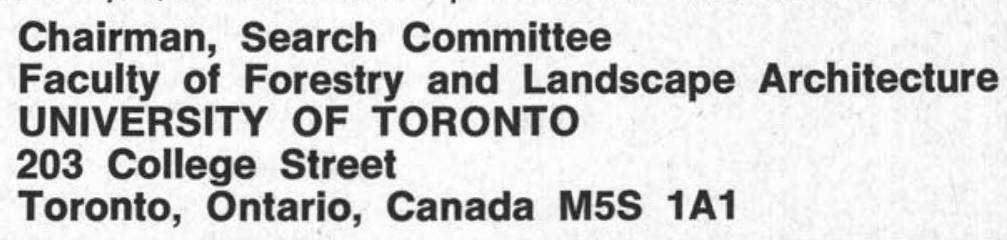

Chairman, Search Committee

Faculty of Forestry and Landscape Architecture

UNIVERSITY OF TORONTO

203 College Street

Toronto, Ontario, Canada M5S 1 A1 